\title{
Woher, wie viel, wie weiter?
}

\author{
Produktion und Konsum von Lebensmitteln wirken auf die Umwelt. Stoffstrom- \\ analysen können die Wege einzelner Produkte von der Rohstoffentnahme bis \\ zur Entsorgung aufzeigen und ganzheitlich die Folgen für Umwelt, Kosten und \\ Beschäftigung abschätzen. Es gibt bereits eine ganze Reihe Untersuchungen, \\ die verschiedene Herstellungswege einzelner Lebensmittel sowie ganze \\ Warenkörbe miteinander vergleichen.
}

Von Ulrike Eberle,

$\mathrm{D}$ Uwe R. Fritsche und Kirstin Wiegmann ie Frage nach künftigen Umweltbelastungen durch veränderte Ernährungsgewohnheiten wurde hingegen bislang unzureichend beantwortet. WissenschaftlerInnen des Öko-Instituts haben im Projekt Ernährungswende nun erstmals die Folgen der künftigen Ernährungsentwicklungen und die von unterschiedlichen Ernährungsstilen untersucht.

Die stoffstrombezogenen Berechnungen im Rahmen des Projekts Ernährungswende geben Auskunft über ökologische Folgen sowie über stoffbezogene Risiken und ökonomische Aspekte der Ernährung in Deutschland.

\section{- Ursprung und Verlauf von Stoffströmen}

Die Stoffstromanalyse dient zur produkt- und branchenübergreifenden Darstellung der Stoffund Energieströme inklusive der Transporte sowie deren Umwelteffekte. Die Stoffstromanalyse des Bedürfnisfelds „Ernährung“ blickt sehr umfassend auf das System Ernährung, indem sie die folgenden Aktivitäten einbezieht:

- Anbau und Verarbeitung von Lebensmitteln im In- und Ausland inklusive Futtermittel, Düngung, Transporten, Verpackung, usw.,

- Logistik des Lebensmittelhandels und Energiebedarf der Verkaufsstätten für Kühlung, Beleuchtung usw.,

- Personenverkehr für Einkauf und Außer-HausEssen,

- Energiebedarf für Kühlen, Kochen, Abwasch usw. sowohl in den Haushalten als auch AußerHaus, jeweils inklusive der Geräteherstellung. Vernachlässigt wurden in der Analyse aufgrund geringer Umweltbedeutung nur die ernährungsbedingten Abfälle und der Eigenanbau von Lebensmitteln wie Gemüse aus dem Garten. bensmitteln was Anbau, Verarbeitung, Gütertransport umfasst und dem anteiligen Energiebedarf für die Zubereitung der Lebensmittel wie Kühlen und Kochen. Die Personentransporte für den Lebensmitteleinkauf, Fahrten zum Essen außer Haus spielen dagegen nur eine geringe Rolle.

Nach dem REF-Szenario werden die Treibhausgasemissionen des Inner-Haus-Verzehrs (IHV) bis zum Jahr 2030 leicht rückläufig sein, während die des Außer-Haus-Verzehrs (AHV) ansteigen.

In der Summe wird die Gesamtemission pro Haushalt - unter anderem durch gestiegene Energieund Fahrzeugeffizienzen - bis 2030 leicht reduziert werden können. Klima- und Ressourcenschutzziele werden damit allerdings nicht erreicht, nur der Status Quo kann gesichert werden.

Stoffstromanalysen beschreiben nicht nur, was heute ist, sondern sind zukunftsoffen. Sie verwenden Szenarien, keine Prognosen, die über Wenn-Dann-Aussagen die Umwelt- und Kostenauswirkungen künftiger Entwicklungsmöglichkeiten beleuchten.

Als Messlatte dient dabei meist die heutige Situation (IST) - hierfür wurde das Basisjahr 2000 genutzt. Demgegenüber stellt das Referenz-Szenario (REF) über eine Trendentwicklung die Situation im Jahr 2030 dar und nimmt an, dass bis dahin keine nennenswerten politischen Maßnahmen zur Veränderung der Ernährung ergriffen werden.

Die folgende Abbildung zeigt die vorläufigen Ergebnisse für die Situationen im Jahr 2000 (IST) und die des Referenz-Szenarios im Jahr 2030 (REF) hinsichtlich der Verursachung von klimawirksamen Schadstoffen. Sie enthält Angaben für den Durchschnittshaushalt, inklusive vorgelagerter Prozessketten und Materialvorleistungen im In- und Ausland, ohne Herstellung, Gebäude/ Wohnungen. IHV steht für Inner-Haus-Verzehr, AHV steht für Außer-Haus-Verzehr.

Die Treibhausgasemissionen der Ernährung resultieren sowohl heute als auch in Zukunft zu jeweils knapp der Hälfte aus der Bereitstellung von Le-

\section{Varianten der Zukunft}

Die Darstellung der Referenzentwicklung ist der erste Schritt - sie beschreibt ja nur den eingriffslosen Trend. Im weiteren Projektverlauf werden noch andere Szenarien erstellt, die Varianten des Trends untersuchen und zusammen den so genannten Szenarioraum ergeben, der den potenziellen Handlungskorridor in der Zukunft umreißt.

Die folgende Tabelle führt die Bereiche auf, in denen sich die Ernährung künftig unterschiedlich entwickeln kann. Sie repräsentieren die Dimensionen des Szenarioraums zur Ableitung von Varianten im Ernährungswende-Projekt.

Diese Varianten können auch miteinander kombiniert werden, um kumulative Effekte zu beleuchten. Optional können künftig auch Varianten zu geänderten Lebensmittel-Importen, zur geänderten Lebensmittelverarbeitung sowie Variationen der Transporte entwickelt werden, dies liegt jedoch nicht im Fokus des laufenden Vorhabens.

Das Ernährungswende-Projekt unterteilt zwar seine Untersuchungen des gesellschaftlichen Handlungsfelds Ernährung in einzelne Module,

\section{Abbildung 1: Gegenïberstellung der Treibhausgasemissionen des Bedïrfnisfeldes Ernöhrung}

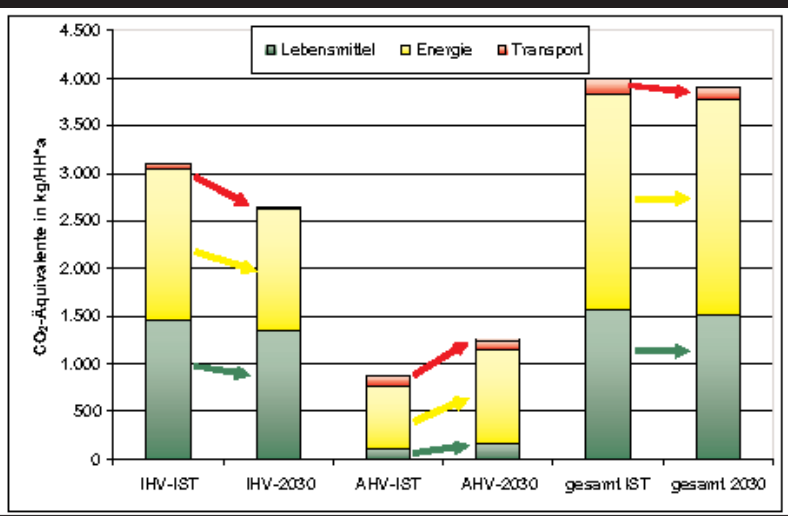


verfolgt aber aufgrund seines sozialökologischen Forschungskonzepts auch den Anspruch der Integration. Damit ist vor allem die Verschränkung und Befruchtung der einzelnen Untersuchungsperspektiven und Problemzugänge zu einer möglichst gemeinsamen Problemsicht zu verstehen.

Diesem Anspruch folgend wurden die vom Institut für sozial-ökologische Forschung erhobenen Ernährungsstile mit Stoffstromdaten unterfuittert, was ihre umweltbezogene Bewertung erlaubt (1). Die empirisch ermittelten Präferenzen der Ernährungsstile für bestimmte Nahrungsmittel wurden in personenbezogene ,Menüs“ übersetzt, mit denen dann der jährliche Pro-Kopf-Verzehr an Lebensmitteln je Ernährungsstil berechnet wurde. Diese Mengen wurden mit den Stoffstromdaten verknüpft und die Lebenswegbilanzen der entsprechenden ,Menüs" ermittelt.

Beispiel: Das Menü des Ernähungsstils Billigund Fleisch-Esser enthält unter anderem 38 Kilogramm Fleisch pro Kopf und Jahr, während im Ernähungsstil der ernährungsbewussten Anspruchsvollen nur 26 Kilogramm Fleisch pro Kopf und Jahr verzehrt werden - dafür aber mehr Gemüse, Teigwaren und Obst. Das folgende Bild zeigt die Ergebnisse der Stoffstromanalyse für Treibhausgasemissionen und versauernde Luftschadstoffe, die heute jeweils mit den Ernährungsstilen verbunden sind. Abgebildet sind ernährungsstilbezogene Angaben pro Person und Jahr, inklusive vor gelagerter Prozessketten wie Kühlung, Transporte und Anbau, Ernte, Verarbeitung, ohne Herstellung von Gebäuden, Wohnungen und ohne Außer-Haus-Verzehr. Die Stoffstromanalyse zeigt, dass der Ernährungsstil der ernährungsbewussten Anspruchsvollen die geringsten Umwelteffekte beim Nahrungsmittelverzehr bewirkt, während sich bei den Billig- und Fleisch-Essern die relativ höchsten Effekte zeigen. Alle anderen Ernährungsstile liegen

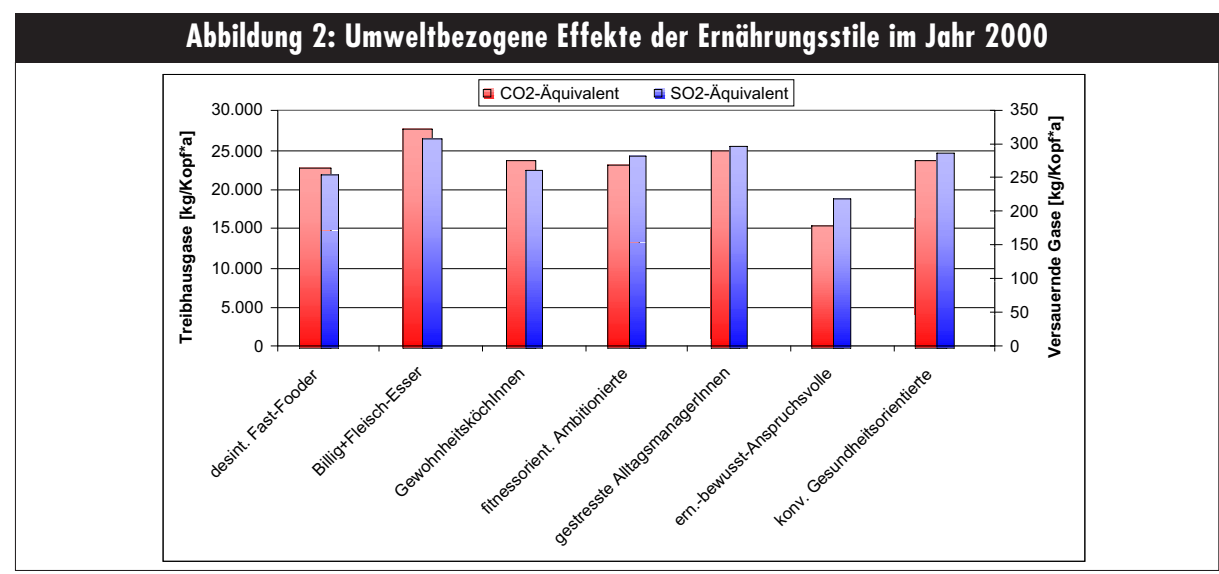

im Rahmen der Datenunsicherheiten praktisch gleich. Überraschend ist auch der Ernährungsstil desinteressierte Fast-Fooder.

Die Billig- und Fleisch-Esser liegen bei den Treibhausgasen um knapp 80 Prozent über dem emissionsärmsten Ernährungsstil der ernährungsbewussten Anspruchsvollen, bei den Schwefeldioxid-Äquivalenten sind es rund 40 Prozent mehr. Die anderen Ernährungsstile liegen gegenüber dem Minimum bei den Treibhausgasen um 50 bis 60 Prozent und bei versauernden Gasen um 20 bis 30 Prozent höher.

Ohne die beiden Extreme ist damit die Trennschärfe der Ernährungsstile gering. Auch der aus Umweltsicht ungünstigste Ernährungsstil liegt absolut gesehen nur knapp über den anderen.

Die im Vergleich zu den anderen Ernährungsstilen geringe Umweltbelastung bei den ernährungsbewussten Anspruchsvollen begründet sich aus zwei Faktoren: dem unterdurchschnittlichen Verzehr von Fleisch und dem überdurchschnittlichen Anteil von Bio-Produkten aus ökologischem Anbau und Tierhaltung.

Bis zum Projektende werden die Ernährungsstile auch noch stoffstromanalytisch in Bezug auf die verschiedenen Anteile von Inner- und AußerHaus-Verzehr hin untersucht - hier werden zum Beispiel andere Zubereitungsformen und Logistik vermutet.

\begin{tabular}{|c|c|}
\hline Variation & Dimension im Szenarioraum \\
\hline Verzehrort & $\begin{array}{l}\text { Inner-Haus- im Gegensatz zu Außer-Haus-Verzehr (Restaurants, Mensen etc.): } \\
\text { stärkere Steigerung von AHV gegenüber dem Referenz-Szenario, anteilige Reduktion IHV }\end{array}$ \\
\hline Produktionsweise & $\begin{array}{l}\text { Steigerung des Anteils von Lebensmitteln aus ökologischer Landwirtschaft im Gegensatz zu } \\
\text { Lebensmitteln aus konventioneller Landwirtschaft: verstärkter Kauf von Bioprodukten zu Lasten } \\
\text { konventioneller Produktion }\end{array}$ \\
\hline Ernährungsweise & $\begin{array}{l}\text { Steigerung des Anteil vegetarischer Ernährung gegenüber Fleischkonsum: Reduktion des } \\
\text { Fleischanteils an der Ernährung zu Gunsten von Gemüse-, Milch- und Getreideprodukten }\end{array}$ \\
\hline Zubereitungsweise & $\begin{array}{l}\text { Reduzierte Steigerung des Anteils der Convenience-Produkte an Lebensmitteln zu Gunsten des } \\
\text { Anteils selbst zubereiteter Lebensmittel }\end{array}$ \\
\hline
\end{tabular}

Bei den Anteilen von Fertigprodukten sind dagegen aus der heutigen Datenlage aus Stoffstromsicht keine nennenswerten Unterschiede zwischen den Ernährungsstilen ableitbar - die „Trennschärfe“ dieser Dimension wird daher in den Szenario-Varianten näher beleuchtet.

Neben der integrativen Analyse von Ernährungsstilen aus Stoffstromsicht werden im weiteren Projektverlauf auch die Ergebnisse des Arbeitspakets „Risiken durch unerwünschte Stoffe und Organismen in Lebensmitteln " in den Stoffstromansatz einbezogen, also Risikofragen integriert. Zudem werden nach Abschluss der Szenario-Arbeiten auch deren ökonomische Wirkungen untersucht, da die Kostenfrage aus Sicht der KonsumentInnen ja keine kleine Rolle spielt - Stichwort: Preisbewusstsein. Für den Sommer 2005 sind somit eine Reihe von Ergebnissen zu erwarten, mit denen die Diskussion zur Ernährungswende aus Sicht von Umwelt und Ökonomie weitergeführt werden kann.

\section{Anmerkung
(1) Vergleiche auch den Artikel von Hayn in diesem Heft. \\ Anmerkung
(1) Vergleiche auch den Artikel von Hayn in diesem Heft.}

Die AutorInnen
Uwe R. Fritsche ist Koordinator, Kirsten Wieg-
mann wissenschaftliche Mitarbeiterin im Bereich
Energie und Klimaschutz im Darmstädter Büro des
Öko-Instituts. Dr. Ulrike Eberle ist stellvertretende
Koordinatorin im Bereich Produkte und Stoffströme
im Freiburger Büro des Öko-Instituts.
Kontakt: Öko-Institut Freiburg, Binzengrün 34a,
79114 Freiburg. Öko-Institut Darmstadt, Rheinstraße
95, 64295 Darmstadt. E-Mail: u.fritsche@oeko.de,
k.wiegmann@oeko.de, v.eberle@oeko.de


(c) 20I0 Authors; licensee IÖW and oekom verlag. This is an article distributed under the terms of the Creative Commons Attribution Non-Commercial No Derivates License (http://creativecommons.org/licenses/by-nc-nd/3.o/), which permits unrestricted use, distribution, and reproduction in any medium, provided the original work is properly cited. 